Revista Brasileira de Agricultura Irrigada v.10, nº.5, p. 965 - 975, 2016

ISSN 1982-7679 (On-line)

Fortaleza, CE, INOVAGRI - http://www.inovagri.org.br

DOI: $10.7127 /$ rbai.v10n500477

Protocolo 477.16 - 01/09/2016 Aprovado em 11/10/2016

\title{
ANÁLISE COMPARATIVA DE METODOLOGIA DE COLETA DE DADOS PARA AVALIAÇÃO DE SISTEMAS DE IRRIGAÇÃO LOCALIZADA
}

\author{
Manoel Valnir Júnior ${ }^{1}$, João Paulo Alves Rocha ${ }^{2}$, Francisco Levy Lima Demontiêzo ${ }^{2}$, Lívia \\ Sales de Sousa Lima ${ }^{3}$, Clayton Moura de Carvalho ${ }^{4}$, Leonaria Luna Silva de Carvalho ${ }^{5}$
}

\section{RESUMO}

Em certas situações, a avaliação de 32 ou mais emissores por setor de irrigação pode ser muito laboriosa, sendo possível que a medição em apenas 16 pontos seja suficiente para a determinação da uniformidade de aplicação de água pelo sistema. Diante disso, objetivou-se com esse trabalho propor uma metodologia a ser usada no campo para determinação da uniformidade de irrigação de sistemas de irrigação localizada de pequeno porte. O sistema de irrigação avaliado estava instalado em um lote de produção particular, cultivado com a cultura da goiaba, situado no Distrito de Irrigação do Baixo Acaraú pertencente ao Departamento Nacional de Obras Contra a Seca. Para a avaliação da uniformidade de distribuição de água foram utilizadas quatro diferentes metodologias: metodologia padrão (CHRISTIANSEN, 1942), onde consiste a coleta de dados em todos os emissores da área a ser avaliada; metodologia proposta por Denículi et al. (1980), onde consiste a coleta de dados 32 emissores; metodologia proposta por Keller \& Karmeli (1975) onde consiste a coleta de 16 emissores e metodologia proposta por este estudo, onde consiste a coleta de dados de 9 emissores. Os resultados obtidos neste trabalho de campo mostraram que o sistema avaliado apresentou uma baixa eficiência, de acordo com os valores dos coeficientes de uniformidade e de eficiência de aplicação, que se apresentaram bastantes inferiores ao recomendado pela literatura especializada no assunto. $\mathrm{O}$ uso da metodologia proposta neste trabalho mostrou-se aceitável para avaliação de sistemas de irrigação localizada semelhantes ao utilizado neste estudo, ou seja, de pequeno porte.

Palavras-chave: coeficientes de uniformidade, avaliação e manejo de sistemas irrigados, microaspersão, gotejamento.

\footnotetext{
${ }^{1}$ Doutor em Engenharia Agrícola, Professor do Eixo de Recursos Naturais do IFCE/Sobral, e-mail: valnir@ifce.edu.br

${ }^{2}$ Graduando Acadêmico de Tecnologia em Irrigação e Drenagem, IFCE/Sobral, e-mails: j.paulo25ipueis@gmail.com; levyfla@hotmail.com

3 Tecnóloga em Irrigação e Drenagem, IFCE Campus Sobral, e-mail: liviaejose@yahoo.com.br

${ }^{4}$ Doutor em Engenharia Agrícola, Professor Colaborador do PRORH/UFS, e-mail: carvalho_cmc@yahoo.com.br

${ }^{5}$ Mestranda em Engenharia Agrícola, UFC, e-mail: leonarialuna@ hotmail.com
} 


\title{
COMPARATIVE ANALYSIS METHODOLOGY OF COLLECTING DATA FOR IRRIGATION SYSTEMS ASSESSMENT LOCALIZED
}

\begin{abstract}
In certain situations, the evaluation of 32 or more emitters for irrigation sector can be very laborious, it being possible for the measurement at only 16 points is sufficient to determine the uniformity of the water system. The research objective with this work propose a methodology to be used in the field to determine the uniformity of irrigation small localized irrigation systems. The estimated irrigation system was installed on a particular production lot, grown with the culture of guava, located in Acaraú Low Irrigation District belonging to the National Department of Works Against Drought. For the assessment of uniformity of water distribution were used four different methods: standard methodology (Christiansen, 1942), which is the collection of data on all issuers of the area to be assessed; methodology proposed by Denículi et al. (1980), which consists of data collection 32 issuers; methodology proposed by Keller \& Karmeli (1975) which is the collection of 16 issuers and methodology proposed by this study, which is a collection of 9 issuers data. The results of this field work showed that the evaluated system showed low efficiency, according to the values of the coefficients of uniformity and application efficiency, which performed far below the recommended by the specialized literature on the subject. The use of the methodology proposed in this study proved to be acceptable for evaluation of trickle irrigation systems similar to those used in this study, ie small.
\end{abstract}

Keywords: uniformity coefficient, evaluation and management of irrigation systems, micro sprinklers, drip.

\section{INTRODUÇÃO}

Segundo Luna et al. (2013) a agricultura irrigada tem sido uma importante estratégia para otimização da produção de alimentos, promovendo desenvolvimento sustentável no campo, com geração de emprego e renda. Já a disponibilidade de água tem se tornado cada vez mais limitante, devendo ser utilizada de maneira criteriosa e precisa, não só visando a otimização da produtividade e da qualidade final do produto, mas também o uso adequado dos recursos hídricos (BERNARDO et al., 2006; MARTINS et al., 2013).

$\mathrm{O}$ desenvolvimento da agricultura irrigada vem crescendo consideravelmente nos últimos anos em todo o Brasil, principalmente com a fruticultura irrigada na região nordeste, que encontrou, no semiárido, um ambiente propício para essa atividade, ainda que a água seja o principal fator limitante nessas regiões, tanto em termos quantitativos quanto qualitativos. Apesar da preocupante questão que é o elevado consumo e às restrições de disponibilidade de água, a irrigação é uma prática essencial quando se quer obter uma produção de forma uniforme e satisfatória.

A agricultura irrigada é muito importante para o desenvolvimento de uma região, é uma atividade que só é rentável e sustentável se realizada de forma adequada através de técnicas que maximizem a eficiência do uso da terra e da água. Assim o sistema de irrigação por gotejamento pode adequar-se em tais técnicas, pois é aplicada diretamente na região radicular em pequenas intensidades e alta frequência, mantendo o solo próximo a capacidade de campo (RIBEIRO et al., 2010).

$\mathrm{Na}$ agricultura irrigada, é importante a avaliação dos parâmetros que afetam a qualidade da irrigação, especialmente aqueles relacionados à uniformidade de distribuição de água (LEVIEN \& FIGUEIRÊDO, 2013).

Mantovani et al. (2009) afirmaram que a avaliação da irrigação é uma importante etapa para obter as informações relacionadas à eficiência de uso da água do sistema de irrigação, perdas durante a aplicação e uniformidade de distribuição de água, 
funcionamento real do sistema (vazão, pressão, lâmina, entupimento, etc.) e necessidade de manutenção.

Segundo Souza et al. (2006), para se avaliar em que condições o sistema de irrigação está operando, parâmetros de desempenho devem ser definidas com base em determinações de campo, como vazão, uniformidade de aplicação e tempo de irrigação. Um dos principais parâmetros usados na avaliação de um sistema de irrigação é a uniformidade de aplicação de água sobre a área irrigada. Uma baixa uniformidade de distribuição de água reduz a eficiência de aplicação de água e a produtividade.

De acordo com Bralts \& Kesner (1983) e Bralts e Edwards (1986), a avaliação de campo de sistemas de irrigação por gotejamento é importante por vários fatores: primeiro, para que os técnicos responsáveis pelos projetos confirmem se o mesmo está bem dimensionado e se a uniformidade de aplicação de água nas subunidades de irrigação atingiu as especificações desejadas; segundo, para que o técnico possa verificar se o desempenho da subunidade é aceitável para aplicação de produtos químicos; e em terceiro lugar, porque a avaliação é uma importante ferramenta para diagnosticar os problemas das unidades $\mathrm{e}$ subunidades de irrigação.

A avaliação do desempenho de um sistema de irrigação é etapa fundamental antes que qualquer estratégia de manejo de irrigação seja implementada, porque é com base nesses resultados que será possível avaliar e adequar o equipamento e sua utilização, em relação aos requerimentos de água dos cultivos utilizados (BERNARDO et al., 2006). No entanto, a avaliação do desempenho de sistemas de irrigação em áreas cultivadas é uma prática que os agricultores têm dado pouca importância.

A avaliação feita no sistema de irrigação localizada pode evitar problemas como baixa uniformidade e eficiência obtendo-se assim valores de aplicação aceitáveis (CARVALHO et al., 2006).

Segundo Levien \& Figueirêdo (2013), para a avaliação da uniformidade de aplicação da água, é necessária a obtenção do coeficiente de uniformidade (CU). Na bibliografia podem ser encontradas referências a vários métodos propostos para estimar a uniformidade de emissão em campo. Destacam-se entre eles: ASAE EP458, Merriam e Keller (1978), Denículi et al. (1980), Bralts e Kesner (1983) e Awulachew et al. (2009).

A melhoria da uniformidade de um sistema de irrigação é uma das decisões mais importantes para o manejo adequado da água aplicada, pois a água em excesso, além da perda de água, pode carrear nutrientes para zonas do solo não exploráveis pelas raízes (BERNARDO et al., 2006). No caso de aplicação em déficit podem ocorrer prejuízos na produção, principalmente nos períodos críticos (SILVA et al., 2008).

A uniformidade de aplicação de água é um parâmetro que caracteriza o sistema de irrigação em função da diferença de volume aplicado na planta ao longo das linhas laterais. A uniformidade da irrigação tem efeito direto no rendimento de culturas, por isso, é considerada como um dos fatores mais importantes no dimensionamento e na operação de sistemas de irrigação (BARRETO FILHO et al., 2000; VALNIR JÚNIOR et al., 2011; FERNANDES et al., 2012).

Segundo López et al. (1992), a determinação do coeficiente de uniformidade (CU) com 16 emissores, previamente selecionados, impede a aplicação de critérios estatísticos e, consequentemente, a definição dos limites de confiança para uma determinada probabilidade. Este total de plantas poderá ser ou não suficiente, em função dos valores reais do coeficiente de uniformidade (CU). Quanto mais baixo o valor do coeficiente de uniformidade (CU), maior deverá ser o número de observações realizadas. Todavia é comprovado que, a partir de 24 observações, os valores reais do coeficiente de uniformidade (CU) praticamente não variam. Em certas situações, a avaliação de 32 ou mais gotejadores por setor pode ser muito laboriosa, sendo possível que a medição em apenas 16 pontos seja suficiente para a determinação da uniformidade de aplicação de água pelo sistema.

Diante disso, objetivou-se com esse trabalho propor uma metodologia a ser usada, no campo, por agricultores e técnicos para 
determinação da uniformidade de irrigação de sistemas de irrigação localizada de pequeno porte, utilizando-se como referência as metodologias padrão (CHRISTIANSEN, 1942), de Keller \& Karmeli (1975) e de Deniculi et al. (1980).

\section{MATERIAL E MÉTODOS}

A coleta de dados (vazão) foi realizada em um lote de produção particular, cultivado com a cultura da goiaba, situado no Distrito de Irrigação do Baixo Acaraú - DIBAU pertencente ao Departamento Nacional de Obras Contra a Seca - DNOCS. O referido Distrito de Irrigação encontra-se na região norte do Estado do Ceará, no trecho final da bacia do Rio Acaraú, abrangendo áreas dos municípios de Acaraú, Bela Cruz e Marco, distando 220 km de Fortaleza, cujo clima da região é o Aw Tropical Chuvoso com precipitação média anual de 900 $\mathrm{mm}$, temperatura média anual de $28,1{ }^{\circ} \mathrm{C}$ e Evaporação média anual de $1.600 \mathrm{~mm}$ (DNOCS, 2004). O Espaçamento utilizado foi de $6 \mathrm{~m} \times 5 \mathrm{~m}$ com um microaspersor por planta.

O sistema avaliado era composto de 10 linhas de irrigação com 15 emissores cada, com espaçamento de $6 \mathrm{~m} \times 5 \mathrm{~m}$, totalizando 150 emissores com aproximadamente 2 anos de funcionamento. $\mathrm{O}$ emissor utilizado foi do tipo Netafin Supernet autocompensante com vazão de $40 \mathrm{~L} \mathrm{~h}^{-1}$.

$\mathrm{Na}$ medição de vazão, foram selecionados emissores representativos de acordo com cada metodologia estudada neste

A.

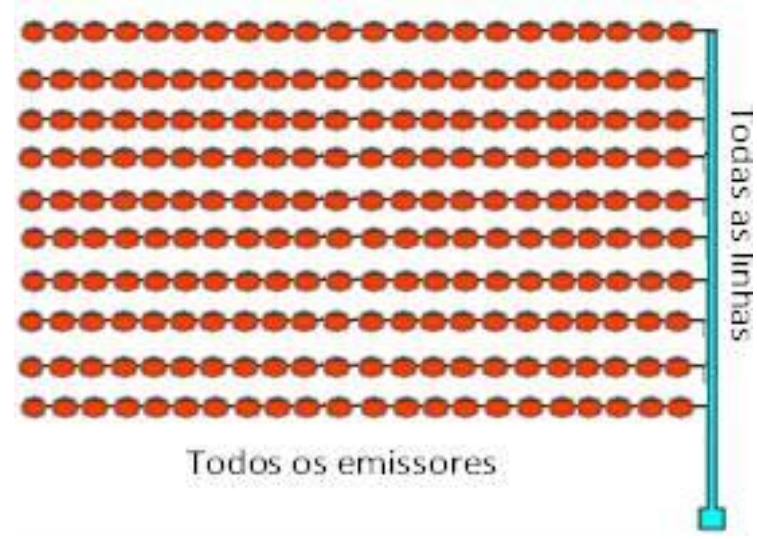

trabalho de campo e coletado um volume de água, utilizando um recipiente de coleta e uma proveta de $500 \mathrm{~mL}$, aplicado em um período de tempo determinado com cronômetro, sendo utilizado o número de três repetições por cada emissor. Os dados coletados em $\mathrm{mL}$ foram anotados em planilhas de campo, transformados em $\left(\mathrm{L} \mathrm{h}^{-1}\right)$ e processados para determinação dos coeficientes de uniformidade.

Para a avaliação da uniformidade de distribuição de água foram utilizadas quatro diferentes metodologias: a) metodologia padrão (CHRISTIANSEN, 1942), onde consiste a coleta de dados em todos os emissores da área a ser avaliada, ou seja, nos 150 emissores (Figura 1A); b) metodologia proposta por Denículi et al. (1980), onde consiste a coleta de dados em quatro linhas laterais (primeira linha, linha situada a $1 / 3$ da origem, linha situada a $2 / 3$ da origem e última linha) e oito emissores por linha (primeiro emissor; emissores a 1/7, 2/7, 3/7, 4/7, $5 / 7$ e 6/7 da origem; e último emissor), totalizando assim 32 emissores (Figura 1B); c) metodologia proposta por Keller \& Karmeli (1975) onde consiste a coleta de dados em quatro linhas laterais (primeira linha, linha situada a $1 / 3$ da origem, linha situada a $2 / 3$ da origem e última linha) e quatro emissores por linha (primeiro emissor; emissores a 1/3, 2/3 e último emissor), totalizando assim 16 emissores (Figura 1C) e; d) metodologia proposta por este estudo, onde consiste a coleta de dados em três linhas laterais (primeira linha, linha central e última linha) e três emissores por linha (primeiro emissor; emissores central e último emissor), totalizando assim 9 emissores (Figura 1D).

B.

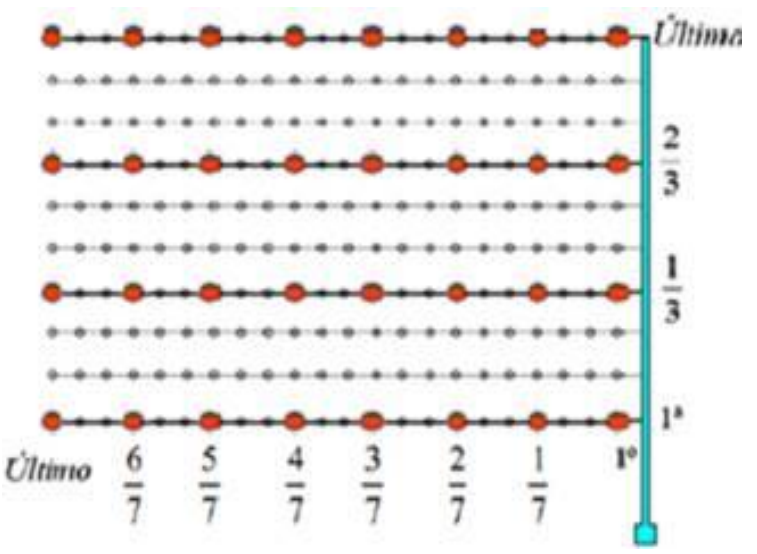


C.

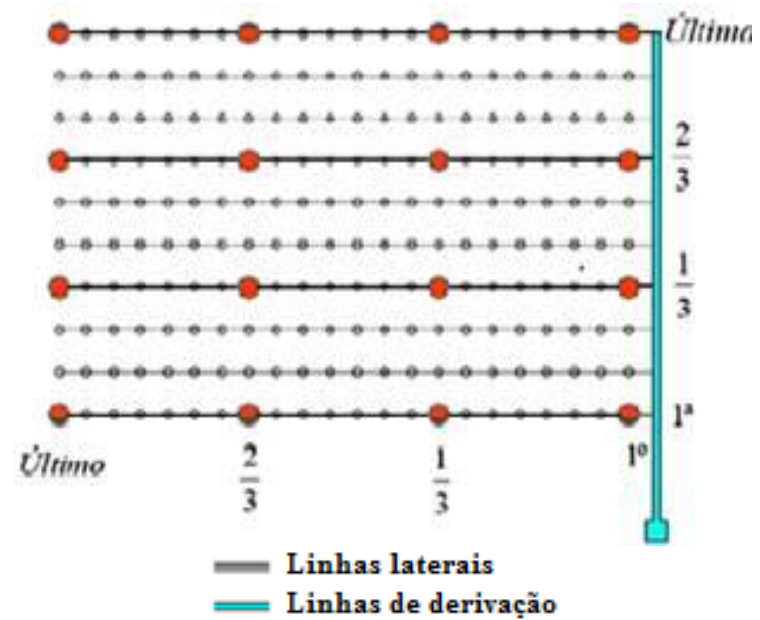

D.

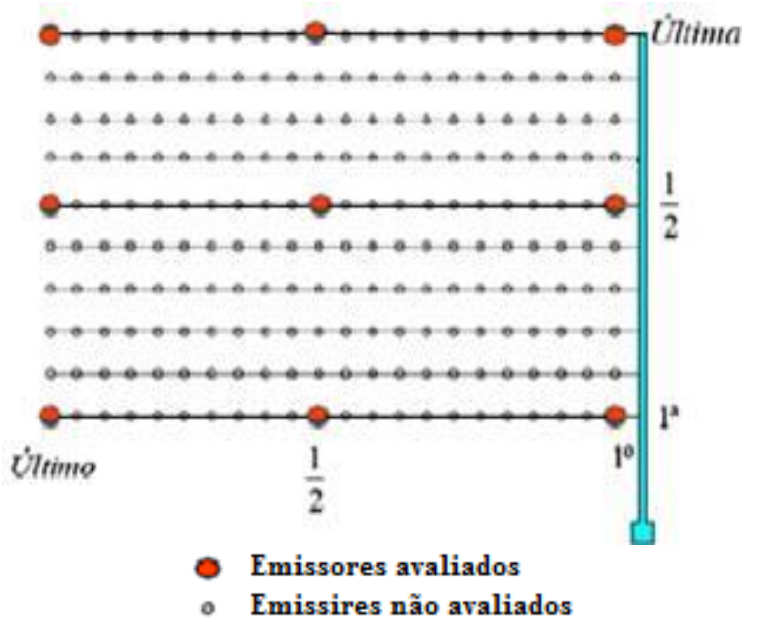

Figura 1. Esquema de seleção de pontos para coletas de dados pelas metodologias:padrão (A), Denículi et al. (B), Keller \& Karmeli (C) e proposto (D).

Com os dados coletados foram calculados: Coeficiente de Uniformidade de Distribuição (CUD), Coeficiente de Uniformidade Absoluto (Cua), Uniformidade Estatística (Us) e Eficiência de Aplicação (Ea), empregando-se as Equações 1, 2, 3 e 4, respectivamente.

$$
\mathrm{CUD}=\frac{\mathrm{Qn}}{\mathrm{Qa}} \times 100
$$

Onde: Qn é a média das $25 \%$ menores vazões dos emissores, em $\mathrm{L} \mathrm{h}^{-1}$; Qa é a média das vazões de todos os emissores avaliados, em $\mathrm{L} \mathrm{h}^{-1}$. $\mathrm{Na}$ utilização da metodologia proposta por este trabalho alterou-se o valor de Qn para a média das 33,33\% menores vazões dos emissores, em $\mathrm{Lh}^{-1}$.

$$
\text { CUa }=9,37365+\operatorname{CUD} \times 0,88840
$$

Onde: CUa é o coeficientes de uniformidade absoluto; CUD é o coeficiente de uniformidade

\section{RESULTADOS E DISCUSSÃO}

Com os dados obtidos em campo, observase na Tabela 1 que a média das de distribuição. Esta é uma equação de correlação proposta por Favetta et al. (1993) sendo utilizada neste estudo por sua praticidade e rapidez em obtenção dos dados a partir de um único coeficiente de uniformidade.

$$
\text { Us }=27,41242+\text { CUD } \times 0,72094
$$

Onde: Us é a uniformidade estatística; CUD é o coeficiente de uniformidade de distribuição. Esta é uma equação de correlação proposta por Favetta et al. (1993) sendo utilizada neste estudo por sua praticidade e rapidez em obtenção dos dados a partir de um único coeficiente de uniformidade.

$$
\mathrm{Ea}=\mathrm{Ks} \times \mathrm{CUD}
$$

Onde: Ks é o coeficiente de transmissividade, para este estudo utilizou-se o valor de $90 \%$, sendo que o Ks desejável está em torno de 85 a 90\% segundo Vermeiren \& Jobling (1997).

vazões coletadas nas diferentes metodologias de coletas de dados encontram-se próximas sem variação expressiva entre elas.

Tabela 1. Valores obtidos com a avaliação em campo.

\begin{tabular}{llll}
\hline \multicolumn{4}{c}{ Valores Obtidos com diferentes metodologias de coleta de dados } \\
\hline Padrão & Keller \& Karmeli (1974) & Denículi et al. (1980) & Proposto \\
\hline
\end{tabular}




\begin{tabular}{ccccc}
\hline Qa $\left(\mathrm{L} \cdot \mathrm{h}^{-1}\right)$ & 26,85 & 27,68 & 26,35 & 27,43 \\
Qn $\left(\mathrm{L} \cdot \mathrm{h}^{-1}\right)$ & 23,10 & 21,84 & 20,94 & 21,40 \\
CUD $(\%)$ & 86,04 & 78,92 & 79,46 & 78,01 \\
CUa*(\%) & 85,81 & 79,48 & 79,97 & 78,68 \\
Us* $(\%)$ & 89,44 & 84,31 & 84,70 & 83,65 \\
Ea $(\%)$ & 77,43 & 71,02 & 71,51 & 70,21 \\
\hline
\end{tabular}

Qa - média das vazões de todos os emissores, Qn - média das 25\% menores vazões dos emissores, CUD - Coeficiente de Uniformidade de distribuição, CUa - Coeficiente de uniformidade absoluto, Us - uniformidade estatística, Ea - eficiência de aplicação. *valores obtidos através de equação de correção propostas por Faveta et al. (1993) e validadas por Carvalho et al. (2006).

\section{Vazões médias do sistema}

Verifica-se na Tabela 1 que para as metodologias estudadas neste trabalho a vazão média do sistema encontra-se na faixa de 26,35 $\mathrm{L} \mathrm{h}^{-1}$ (utilizando a metodologia proposta por Denículi et al.) a 27,68 $\mathrm{L} \mathrm{h}^{-1}$ (utilizando a metodologia proposta por Keller \& Karmeli), apresentando um intervalo de variação de 1,33 L. $\mathrm{h}^{-1}$ entre o maior valor e o menor valor das vazões médias encontradas pelas diferentes metodologias de coleta de dados.

Tomando como base a metodologia padrão (CHRISTIANSEN, 1942) onde todos os
150 emissores são avaliados, observa-se que a metodologia proposta por Keller \& Karmeli (16 emissores avaliados) e a metodologia proposta por este trabalho (9 emissores avaliados) superestimam a vazão média do sistema em $3,09 \%$ e $2,16 \%$, respectivamente, e que a metodologia proposta por Denículi et al. subestima o valor em $1,86 \%$, tais variações são mínimas e aceitáveis. A variação utilizando a metodologia proposta encontra-se praticamente na média entre as variações positivas e negativas observadas pelas metodologias de Keller \& Karmeli e Denículi et al., respectivamente (Figura 2).

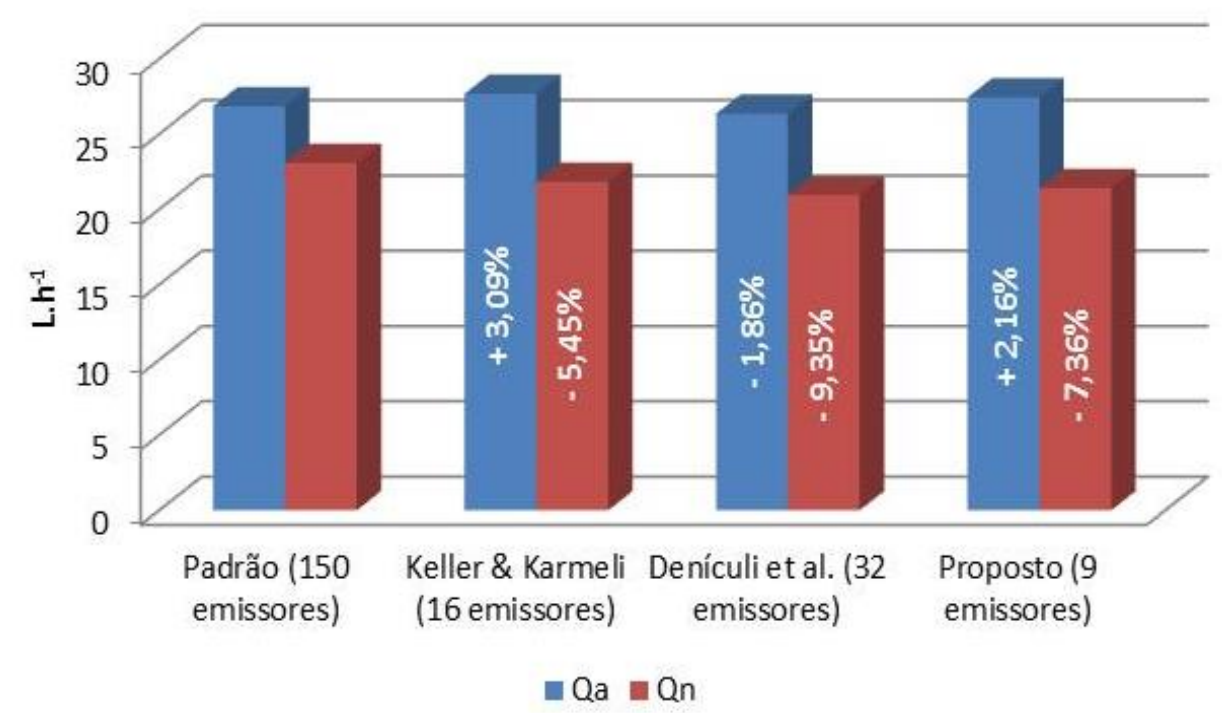

Figura 2. Valores das vazões coletadas em campo por diferentes metodologias.

No que se refere as médias das $25 \%$ menores vazões coletadas em campo verifica-se que todas as metodologias utilizadas neste experimento subestimam o valor encontrado pela metodologia padrão, sendo que a metodologia de Keller \& Karmeli subestima em $5,45 \%$, a metodologia de Denículi et al. em $9,35 \%$ e a metodologia proposta em $7,36 \%$
(Figura 2). Observa-se que o valor encontrado pela metodologia proposta foi praticamente o intervalo médio entre as duas metodologias utilizadas atualmente (Keller \& Karmeli e Denículi et al.).

\section{Coeficiente de Uniformidade de Distribuição (CUD)}


Os valores encontrados do coeficiente de uniformidade de distribuição (CUD) pelas diferentes metodologias de coleta de dados foram iguais a $86,04 \%$ (padrão), 78,92\% (Keller \& Karmeli), 79,46 (Denículi et al.) e 78,01\% (proposto). O valor de
CUD encontrado pelo método padrão é classificado como bom e os valores de CUD encontrados pelas demais metodologias foram classificados como regular de acordo com a classificação de Bralts (1986) citados na Tabela 2.

Tabela 2. Critérios para classificação de CUD e CUa.

\begin{tabular}{cc}
\hline CUD e CUa & Classificação \\
\hline $90 \%$ ou maior & Excelente \\
$80 \%$ a $90 \%$ & Bom \\
$70 \%$ a $80 \%$ & Regular \\
Menor que $70 \%$ & Ruim \\
\hline
\end{tabular}

Bralts (1986)

Observa-se, na Tabela 1, que o valor do CUD obtido na grande maioria das diferentes metodologias de coletas de dados é o menor entre os valores dos demais coeficientes analisados, porém em sistemas de irrigação localizada, segundo López et al. (1992), ele é o mais usado na avaliação, pois este possibilita uma medida mais restrita, dando maior peso às plantas que recebem menos água.

$\mathrm{Na}$ Figura 3 observa-se que o CUD em todas as metodologias utilizadas, exceto a padrão que avalia todos os emissores do sistema de irrigação, encontram-se com valores percentuais muito próximos, demonstrando uma variação entre elas de $1,45 \%$, justificando a aceitação das metodologias de Keller \& Karmeli (16 emissores), de Deniculi et al. (32 emissores) ou a protosta (9 emissores) para o tipo de sistemas de irrigação avaliado neste estudo, sistemas de irrigação de pequeno porte.

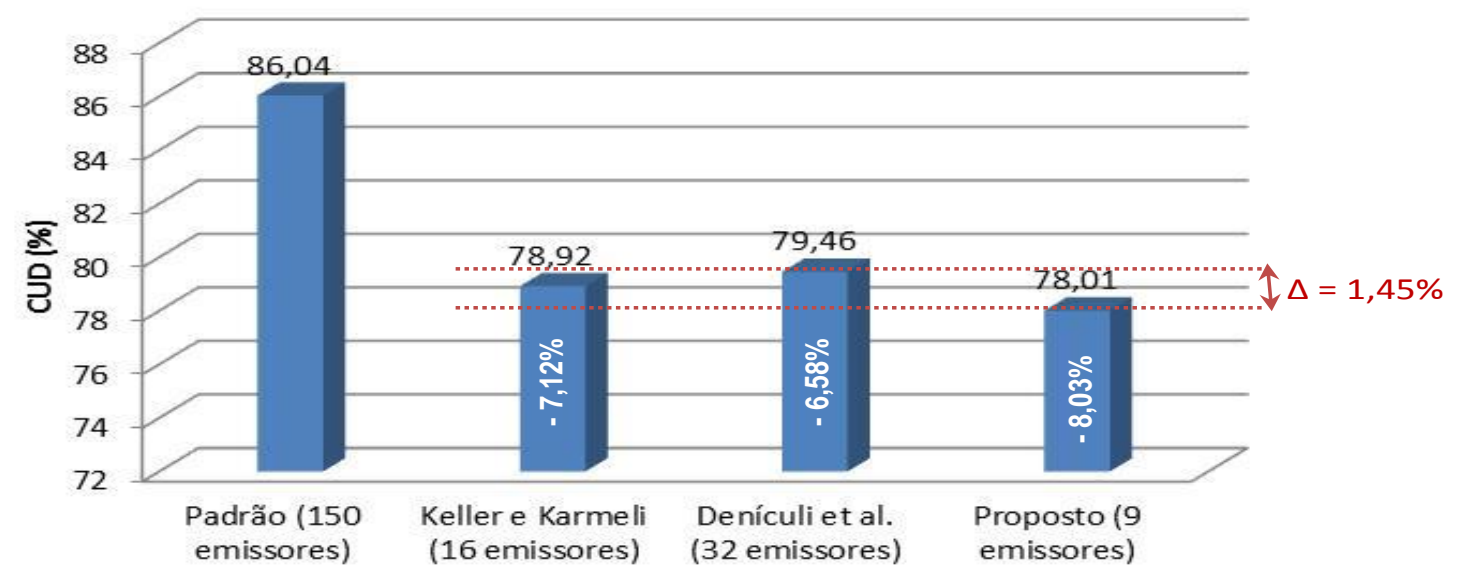

Figura 3. Valores de CUD obtidos de diferentes metodologias de coletadas de dados.

Os resultados obtidos neste estudo corroboram com Ribeiro et al. (2010) que concluíram que a metodologia de amostragem com 16 pontos (Keller \& Karmeli) teve menor uniformidade que a metodologia de amostragem com 32 pontos (Denículi et al.) no sistema de irrigação por gotejamento.
Os valores obtidos encontram-se muito abaixo do recomendado pela literatura para sistemas de irrigação localizada, sendo que este resultado inferior pode ser atribuído ao entupimento dos emissores e vazamentos nas tubulações observados durante a realização das irrigações. Situação semelhante foi encontrada por Carvalho et al. (2006) e Nascimento et al. 
(2009), trabalhando com avaliação de sistemas de irrigação localizada.

\section{Coeficiente de Uniformidade Absoluta (CUa)}

Observa-se na Tabela 1 que os valores encontrados do coeficiente de uniformidade absoluta (CUa) pelas diferentes metodologias de coleta de dados foram iguais a 85,81\% (padrão), 79,48\% (Keller \& Karmeli), $79,97 \%$ (Denículi et al.) e 78,68\% (proposto), comportamento este idêntico ao comportamento apresentado com os valores de CUD na tabela de classificação de Bralts (1986) citados na Tabela 2.

Na Figura 4 observa-se que o valor de CUa nas metodologias mais usuais (Keller \& Karmeli e Denículi et al.) juntamente com a metodologia de coleta de dados proposta neste trabalho de campo, encontram-se com valores muito próximos com uma variação percentual entre eles de apenas $1,29 \%$, justificando assim para sistemas de irrigação de pequeno porte a utilização de qualquer umas das três metodologias para cálculo de Coeficiente de Uniformidade Absoluta.

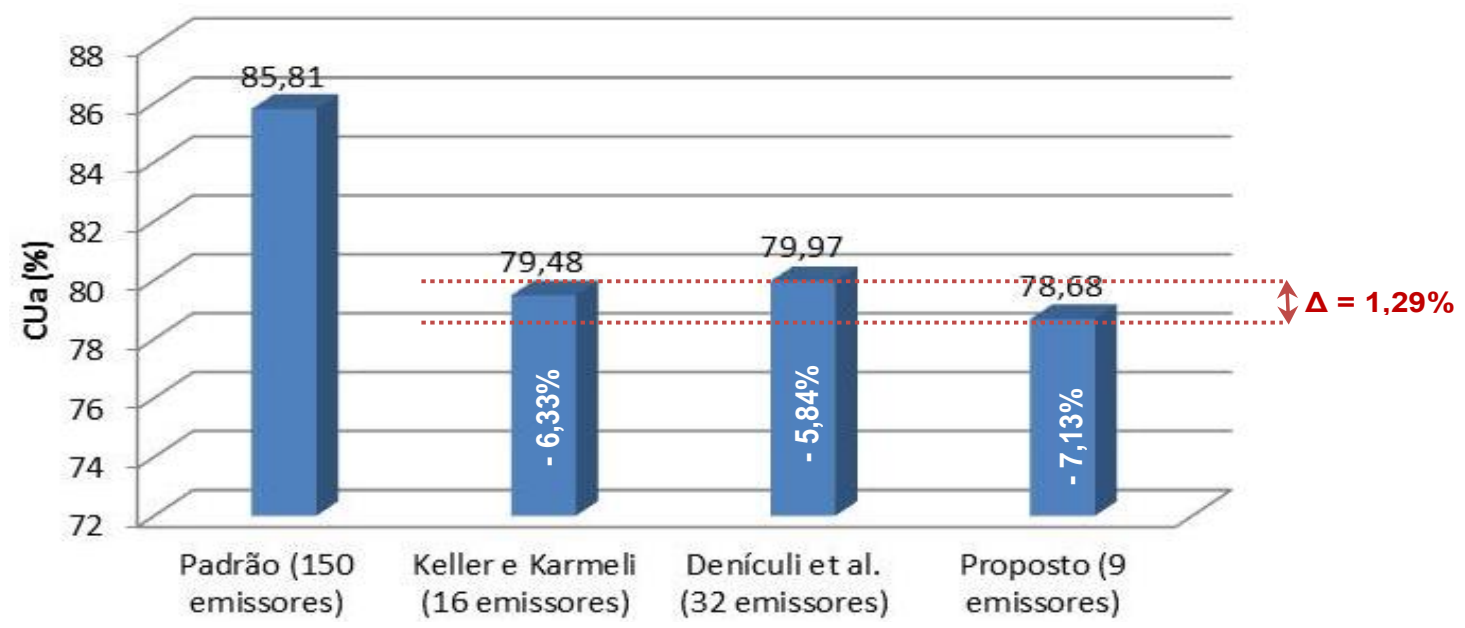

Figura 3. Valores de CUa obtidos de diferentes metodologias de coletadas de dados.

Silva \& Silva (2005) afirmam que em sistemas de irrigação por gotejamento, quanto maior o número de gotejadores avaliados, mais precisos são os valores dos coeficientes de uniformidade, demonstrando que o método de Denículi et al. (1980), apesar de ser um pouco mais trabalhoso, é mais apropriado que o de Keller \& Karmeli (1975).

\section{Uniformidade Estatística (Us)}

Os valores encontrados da uniformidade estatística - Us (Tabela 1) para todas as metodologias de coletas de dados encontram-se entre 80 e $90 \%$, sendo classificados por Favetta $\&$ Brotel (2001) como muito bom.

$\mathrm{Na}$ Figura 5 observa-se que há uma diferença baixíssima nos valores de Us nas metodologias mais usuais (Keller \& Karmeli e Denículi et al.) juntamente com a metodologia de coleta de dados proposta neste trabalho de campo, em torno de $1 \%$. 


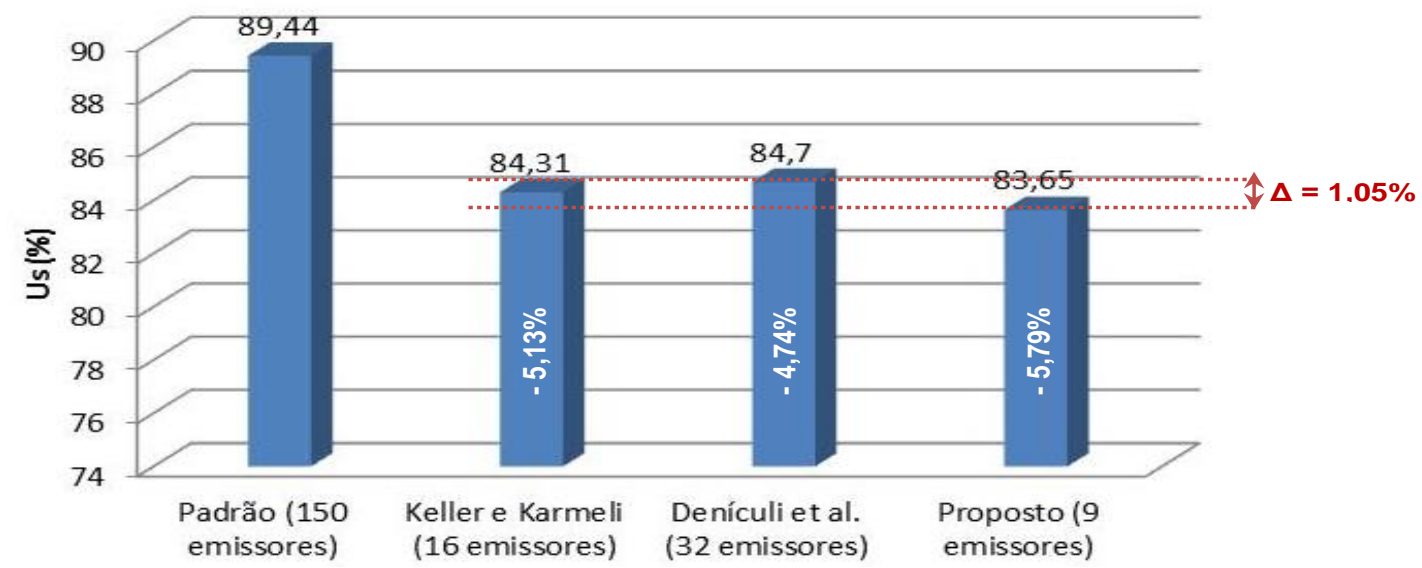

Figura 5. Valores de CUa obtidos de diferentes metodologias de coletadas de dados.

\section{Eficiência de Aplicação (Ea)}

O sistema apresentou uma eficiência de aplicação (Ea) com as diferentes metodologias de coleta de dados inferior ao valor recomendado por Sousa (2003), que deve está entre 90 a $95 \%$.

Os valores observados na Figura 6 indicam uma baixa variação percentual entre as metodologias mais usuais, em torno de $1,30 \%$ e uma variação negativa entre estas metodologias e o método padrão (que avalia todos os emissores do sistema) em torno de 6,55\%. Mesmo assim tomando como base as metodologias mais utilizadas atualmente o método proposto por este trabalho apresenta-se bem confiável e com baixa diferença percentual.

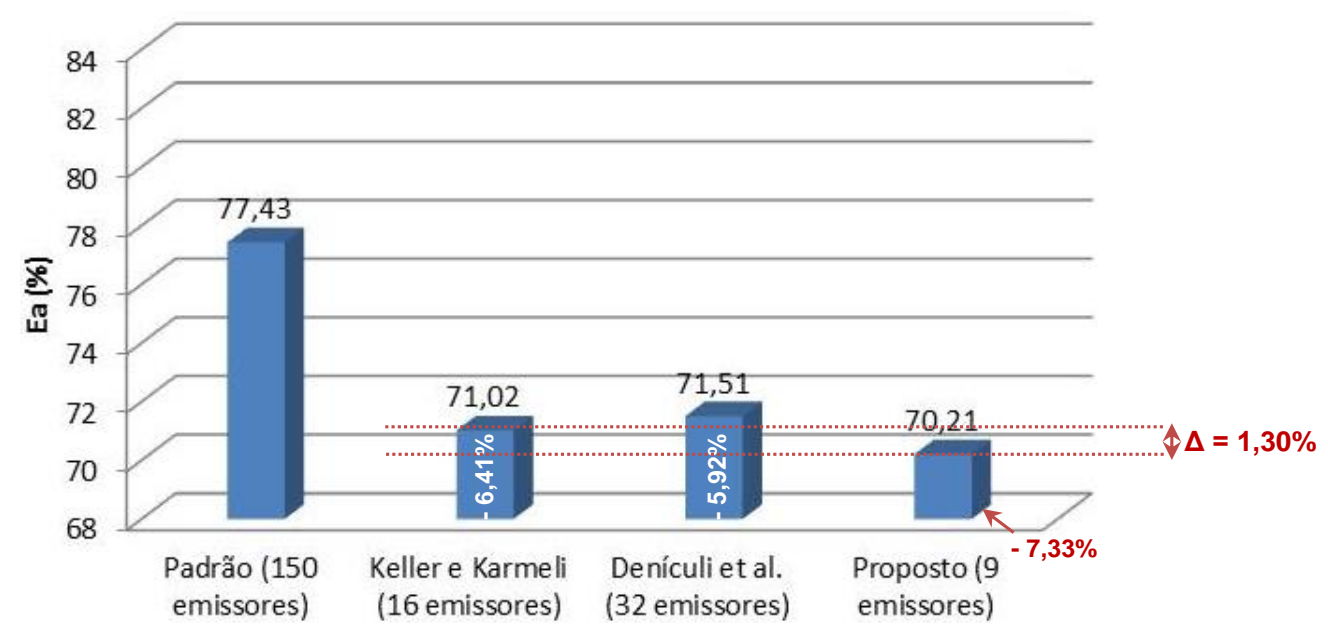

Figura 6. Valores de Ea obtidos de diferentes metodologias de coletadas de dados.

\section{CONCLUSÃO}

Os resultados obtidos neste trabalho de campo mostraram que o sistema avaliado apresentou uma baixa eficiência, de acordo com os valores dos coeficientes de uniformidade e de eficiência de aplicação, que se apresentaram bastantes inferiores ao recomendado pela literatura especializada no assunto.

O uso da metodologia proposta neste trabalho que sugere 9 (nove) pontos de coletadas de dados mostrou-se aceitável para avaliação de sistemas de irrigação localizada semelhantes ao utilizado neste estudo, ou seja, de pequeno porte.

Os valores dos coeficientes de uniformidades não apresentaram diferenças expressivas entre as metodologias propostas por Keller \& Karmeli, Denículi et al. e a proposta neste estudo de campo.

Sugere-se avaliar um maior número e diferentes de portes de sistemas de irrigação localizada utilizando as mesmas metodologias de coleta de dados utilizadas neste estudo para 
uma maior segurança na recomendação da metodologia proposta e confiabilidade dos dados dos coeficientes de uniformidade.

\section{REFERÊNCIAS BIBLIOGRÁFICAS}

ASAE EP458. Field evaluation of microirrigation systems. In: ASAE Standards 1998, St. Joseph: ASAE, 1998. p. 908-914.

AWULACHEW, S. B.; LEMPERIERE, P.; TULU, T. Training manual on agricultural water management. IWMI, ILRI, Adama University, Ethiopia, 2009. 235 p.

BARRETO FILHO, A. de A.; DANTAS NETO, J.; MATOS, J. A. de; GOMES, E. M.; Desempenho de um sistema de irrigação por microaspersão, instalado a nível de campo Revista Brasileira Engenharia Agrícola e Ambiental, v.4, n.3, p.309-314, 2000.

BERNARDO, S.; SOARES, A. A.; MANTOVANI, E. C. Manual de irrigação. 8. ed. Viçosa, MG: UFV, 2006. 625p.

BRALTS, V. F. Field performance and evaluation. In: NAKAYAMA, F. S.; BUCKS, D. A. (Ed.) Trickle irrigation for crop production. Amsterdam: Elsevier, 1986. p.216240. (Development in Agricultural Engineering, 9).

BRALTS, V. F.; EDWARDS, D. M. Field evaluation of drip irrigation submain units. Transactions of the ASAE, v. 29, n. 6, p. 16591664, 1986.

BRALTS, V. F.; KESNER, C. D. Drip irrigation field uniformity estimation. Transactions of the ASAE, v. 26, n. 5, p. 1369-1374, 1983.

CARVALHO, C. M. de, ELOI, W. M., LIMA, S. C. R. V., PEREIRA, J. M. G. Desempenho de um sistema de irrigação por gotejamento na cultura da goiaba. Irriga, v.11, p.36-46, 2006.
CHRISTIANSEN, J. E. Irrigation by sprinkling. Berkeley: University of Califórnia, 1942. $124 \mathrm{p}$.

DENICULI, W.; BERNARDO, S.; THIÉBAUT, J. T. L.; SEDIYAMA; G. C. Uniformidade de distribuição de água, em condições de campo, num sistema de irrigação por gotejamento. Revista Ceres, v. 27, n. 150, p. 155-162, 1980.

DNOCS - Departamento Nacional de Obras Contra as Secas. Perímetro de Irrigação Baixo Acaraú. Disponível em: <http://www. dnocs.gov.br/ppi/ce/baixo_acarau.html>.

Acessado em 24/08/2016. 2004.

FAVETTA, G. M.; BOTREL, T. A.; FRIZZONE, J. A. Correlação entre três métodos de estimativa da uniformidade de distribuição em irrigação localizada. Engenharia Rural, Botucatu, v. 4, p. 117-134, 1993.

FERNANDES, A. I.; CARVALHO, M. A. R.; CARVALHO, L. C. C.; SANTOS NETO, A. M. Avaliação de um sistema de irrigação pelas metodologias de Keller e Karmeli, e de Denículi em citros irrigados por gotejamento. Revista Brasileira de Agricultura Irrigada, v.6, n.2, p.74-80, 2012.

KELLER, J.; KARMELI, D. Trickle irrigation design. S.1: Rain Bird Sprinkler Manufacturing Corporation, 1975. $133 \mathrm{p}$.

LEVIEN, S. L. A.; FIGUEIRÊDO, V. B. Metodologia simplificada para a estimativa em campo da uniformidade de sistemas de irrigação por gotejamento superficial. Revista Brasileira de Agricultura Irrigada, v.7, n.5, p.290-299, 2013. DOI:10.7127/rbai.v7n500179

LÓPEZ, J. R.; ABREU, J. M. H.; REGALADO, A. P., HERNANDEZ, J. F. G. Riego localizado. Madrid: MAPA-YRIDA, Ediciones MundiPrensa, 1992. 405 p.

LUNA, N. R. S.; ANDRADE, E. M.; CRISÓSTOMO, L. A.; MEIRELES, A. C. M.; AQUINO, D. N. Dinâmica do nitrato e cloreto 
no solo e a qualidade das águas subterrâneas do distrito de irrigação Baixo Acaraú, CE. Revista Agro@mbiente Online, v.7, n.1, p.53-62, 2013.

MANTOVANI, E. C.; BERNARDO, S.; PALARETTI, L. F. Irrigação: princípios e métodos. 2. ed., atual e ampliado. Viçosa, MG: UFV, 2009. p. 355.

MARTINS, C. L.; BUSATO, C.; SILVA, S. F.; RODRIGUES, W. N.; REIS, E. F. Avaliação do desempenho de sistemas de irrigação no sul do Estado do Espírito Santo. Revista Agro@mbiente On-line, v. 7, n. 2, p. 236-241, 2013.

MERRIAM, J. L., KELLER, J. Farm irrigation system evaluation: a guide for management. 3 ed. Logan: Utah State University, 1978. 271 p.

RIBEIRO, M. D.; KLEIN, M. R.; SZEKUT, F. D.; PALHARI JÚNIOR, M.; VILAS BOAS, M. A. Uniformidade de um sistema de irrigação familiar na cultura do morango por meio de $16 \mathrm{e}$ 32 pontos de amostragem. In: Encontro Anual de Iniciação Científica, 29., 2010, Guarapuava. Resumos... Guarapuava: UNICENTRO, 2010.

SILVA, A. C.; TEODORO, F. E. R.; MELO, B. Produtividade e rendimento do cafeeiro submetido a lâminas de irrigação. Pesquisa Agropecuária Brasileira, v.43, n.3, p.387394, 2008.

SILVA, C. A.; SILVA, C. J. Avaliação de uniformidade em sistemas de irrigação localizada. Revista Científica Eletrônica de Agronomia, Ano.4, n.8, 2005.

SOUZA, L. O. C.; MANTOVANI, E. C.; SOARES, A. A.; RAMOS, M. M.; FREITAS, P. S. L. Avaliação de sistemas de irrigação por gotejamento, utilizados na cafeicultura. Revista Brasileira de Engenharia Agrícola e Ambiental, v.10, n.3, p.541-548, 2006.

VALNIR JÚNIOR, M., CARVALHO, C. M.; SANTOS NETO, A. M.; SOARES, J. I.; LIMA, S. C. R. V.; CARVALHO, M. A. R. Análise de desempenho em laboratório de linha gotejadora antes e após sua utilização em campo. Revista Brasileira de Agricultura Irrigada, v.5, n. 4, p.351-360, 2011.

VERMEIREN, L.; JOBLING. G. A. Irrigação Localizada. Tradução de GHEY, H. R.; DAMASCENO, F. A. V.; SILVA JÚNIOR, L. G. A.; MEDEIROS, J. F. de. Campina Grande. UFPB. 1997. 184 p. (Estudos FAO: Irrigação e Drenagem, 6). 